treatment. Scoring systems, such as the Kobayashi, for risk of CAA despite immunoglobulin (IVIG), do not apply in the UK so we wished to establish our own. This would be useful for patients who were at high risk of IVIG resistance and in whom additional therapy such as corticosteroids could be considered.

Methods We investigated the correlation of biomarkers with CAA in a defined population. Our department will see all children across the region with $\mathrm{KD}$, for a non-selective population of $5.6 \mathrm{~m}$. Any from outside our region were excluded. All those presenting over a 5 year period from August 2012 July 2017 were included in the study. Demographic data and clinical data were recorded for sex, age at diagnosis, clinical features of $\mathrm{KD}$ and laboratory parameters (full blood count, CRP, liver function tests, albumin, and sodium).

Results 90 children were seen (with no case selection) during this time period. Age inversely correlated with CAA development, when those with CAA were compared with those without CAA; mean (SD) in days 823 (804) vs 1378 (970), $\mathrm{p}=0.005 \%$ and $72 \%$ under 6 months had atypical KD. Only four laboratory markers at presentation correlated with CAA development: lower platelets 404.6 (179.6) vs 519.5 $(265.6) \times 10^{9} / \mathrm{L}, \mathrm{p}=0.018$, higher neutrophils 8.23 (8.04) vs $7.8(5.73) \times 10^{6} / \mathrm{L}, \mathrm{p}=0.006$, higher lymphocytes $4.4(2.22)$ vs $\left.3.3(1.74) \times 10^{6} / \mathrm{L}, \mathrm{p}=0.02\right)$ and higher CRP 141 (91) vs 92 (88) $\mathrm{mg} / \mathrm{L}, \mathrm{p}=0.017$. We found that serum sodium did not correlate across both groups 136.2 (4.1) vs 134.0 (18.75) $\mathrm{mmol} / \mathrm{L}, \mathrm{p}=0.533$.

Conclusions It is most important to note that young age, especially those under 6 months of age and in the presence of atypical $\mathrm{KD}$ is highly associated with CAA. In addition, analysis of blood markers shows those who are most likely to benefit from additional therapy at presentation in order to reduce the high risk of CAA in the young UK population.

\section{G87(P) A RETROSPECTIVE REVIEW OF DEATHS IN TRISOMY 21: A TERTIARY CENTRE'S EXPERIENCE SINCE THE MILLENNIUM}

P Shires, E Marder, H Vyas. Nottingham Children's Hospital, Nottingham University Hospitals NHS Trust, Nottingham, UK

\subsection{6/archdischild-2018-rcpch.85}

Aims Children with Trisomy 21 often have complex health needs and are at increased risk of mortality than age-matched peers. A retrospective review was undertaken of children with Trisomy 21 who had died at our tertiary centre since the millennium. We audited demographics, cause of death and preceding events to identify any themes.

Methods A retrospective review of electronic, paper and archived microfilm patient records was undertaken in those with a diagnosis of Trisomy 21 who died in our trust after the millennium.

Results 16 cases were identified; the mean age at death was 34 months (ranging 2 days - 15 years). 50\% of deaths occurred within the first year of life. Of the 13 cases where a cause of death was identified, cardiac pathology was attributed in 2 of 13 cases. Infection was implicated in 9 out of 13 cases, with 7 cases of primarily respiratory illness and 2 cases of line sepsis. Underlying respiratory disease was a significant contributing factor in 4 out of 13 cases. There was 1 case of Trisomy 21 with co-existent lethal skeletal dysplasia and 1 death related to congenital airway abnormality. In the 6 cases where immune function was tested, only one had completely normal function.

Conclusions The burden of cardio-respiratory disease in Trisomy 21 is well recognised. In our experience, sepsis, particularly with respiratory focus, was responsible for a high proportion of deaths. It is important that health care professionals have an awareness of the increased susceptibility and risk of mortality related to sepsis in children with Trisomy 21. The recognised association with impaired immune functioning in Trisomy 21, coupled with underlying cardio-respiratory comorbidities, may heighten susceptibility to mortality in sepsis. To exacerbate this, communication and behavioural difficulties can make assessment more challenging and may mask or impede recognition of the severity of illness. Therefore, it is important for clinicians to be mindful of sepsis and have a low threshold for initiating early and aggressive management including timely administration of antibiotics.

\section{G88(P) IMMUNODEFICIENCY IN CHILDREN WITH DOWN SYNDROME}

D Huggard, M Mahon, F McGrane, N Lagan, C Purcell, J Balfe, E Roche, E Molloy. Academic Paediatrics, Trinity College Dublin, National Children's Hospital, Tallaght, Dublin, Ireland

\subsection{6/archdischild-2018-rcpch.86}

Aims Down Syndrome (DS) is the most common genetic syndrome associated with abnormal immune function and immune defects. There is an increased susceptibility to both bacterial and viral infections. We aimed to examine the degree of immunodeficiencies in children with DS.

Methods Children who attended the specialist multidisciplinary DS clinic in Tallaght were included, and medical details collected especially in relation to infections, recurrent respiratory tract infections (RTIs), hospital admissions and vaccinations. Results of Full blood counts, T and B cell subsets and immunoglobulins were analysed and compared to age specific reference ranges.

Results Twenty-eight children (age range 1-12 years) were included and 16/28 (57\%) had recurrent RTIs. Hospitalisation at least once was necessary in $15 / 28(54 \%)$ patients, and 6/28 (21\%) required multiple admissions. All but one patient's routine immunisations were up to date (96\%). Although 22 children had a normal white cell count (WCC), Neutrophil and lymphocyte levels, $T$ and $B$ cell subsets $(n=13)$ revealed decreased CD3+, Helper T, Cytotoxic T and CD19 + B cells, with the latter being significantly reduced. IgA and IgG levels were normal or high in all cases, and levels were either normal or low for IgM.

Conclusion We found that children with DS were at increased risk of infections, especially recurrent RTIs, with a significant hospitalisation rate. Vaccination compliance was very high, however the CD19 +B cells were found to be low, which may point to a poor memory B cell response. Further research to evaluate individualised vaccination and prophylactic programmes would be valuable in this cohort. 